\title{
Numerical methods for problems with moving interfaces and irregular geometries
}

\author{
Y. F. Yap ${ }^{1} \&$ J. C. Chai ${ }^{2}$ \\ ${ }^{I}$ School of Mechanical and Aerospace Engineering, \\ Nanyang Technological University, Singapore \\ ${ }^{2}$ Department of Mechanical Engineering, The Petroleum Institute, \\ Abu Dhabi, UAE
}

\begin{abstract}
In this article, we present a unified approach to identify an interface and model the associated interface conditions. Interface includes but is not limited to (1) fluid-fluid interface, (2) fluid-solid interface and (3) physical boundaries and/or internal interfaces. We identify an interface using the distance function. The continuum surface force model is used to model surface tension forces at fluidfluid interfaces. This model is then extended to model surface conditions at fluidsolid interfaces and physical boundaries. Capabilities of the current approach are demonstrated using a few test cases. Comparisons with available solutions show that the current approach produces accurate solutions.
\end{abstract}

Keywords: multiphase flows, particular flows, numerical methods, irregular geometries, finite-volume method.

\section{Introduction}

Interfaces are encountered in multiphase flow problems. Multiphase here includes but is not limited to problems with (1) fluid-fluid interface and (2) fluidsolid interface. Identification of these types of interfaces using the distance function has been reported by various researchers [1-7]. Once the interface is identified using the distance function, various procedures can be used to model the transport and/or evolution of the interface.

The level-set method [1] has been used to model droplet evolutions [2] and stratified flows [3-5]. The continuum surface force model [8] was used to model the surface tension forces at the fluid-fluid interface. We extended the continuum 
surface force approach to model fluid-solid interfaces with additional surface forces $[6,7]$.

In this article, we further identified another class of interface where we shall specify using the distance function [9]. The interface conditions are then model using an extension of the continuum surface force model. This new type of interface divides an interior space (e.g. inside of a building) from an external environment (Fig. 1(c)) which we shall termed a physical boundary or separates two materials with different properties or differentiates two regions with different sources.

The objectives of this article is to show that all three classes of interfaces have been successfully modelled using this unified distance function identification together with the continuum surface force model (or its extensions).

The remainder of this article is divided into five sections. The basic types of interfaces are discussed in the next section. This is followed by a discussion on the basic idea of modelling these different types of interfaces. A more detailed description of the approaches used in then given in Section 4. Section 5 presents representative problems before we conclude this article with some concluding remarks.

\section{Types of "Interfaces"}

Figure 1 shows three types of "interfaces". These are (1) interface between two fluids (Fig. 1(a)) which we shall referred to as fluid-fluid interface, (2) interface between a solid and its adjacent fluid (Fig. 1(b)) which we shall called a "fluidsolid (to be consistent with earlier terminology)" interface and (3) interface dividing an interior space (e.g. inside of a building) from an external environment (Fig. 1(c)) which we shall termed a physical boundary or interface which separates two materials with different properties or interface which divides two regions with different source.

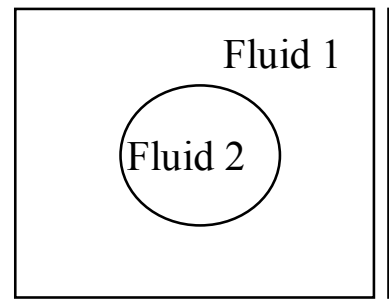

(a)

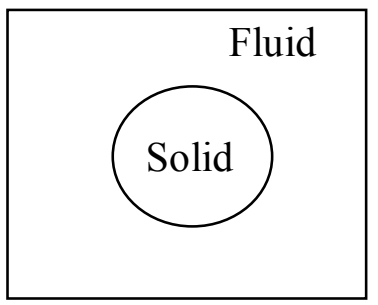

(b)

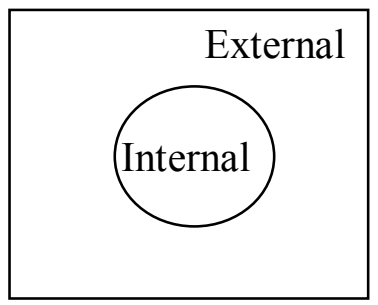

(c)

Figure 1: Three different types of "interfaces", (a) interface between two fluids, (b) interface between a fluid and solid (c) a physical interface separating two environments.

The idea of treating fluid-fluid interface and fluid-solid interface is well known. In this article, we introduce the idea of treating a physical boundary as an 
"interface" similar to a fluid-fluid interface or a fluid-solid interface. We present a unified approach to model the three different types of "interfaces".

For ease of discussion, we shall assume that an interface exist when two media come in contact with each other. The media can be a fluid, a solid and/or an environment (e. g. inside of a building and space external to the building).

At an interface we must capture the interface conditions that exist due to the types of media which come into contact. Surface tension force and Marangoni force are some common forces found at a fluid-fluid interface. Drag force and lift force act on a fluid-solid interface. At a physical boundary, heat can be transferred due to temperature difference between the two "media." In addition to external boundaries, our approach is also capable of capturing internal interfaces which include but are not limited to, (1) interface which separates two materials with different properties, and (2) interface which divides two regions with different source. For ease of discussion and without lost of generality, we shall refer to these as surface "conditions." We shall present a unified approach to treat these three seemingly different surface conditions.

\section{Basic idea}

In this article, we utilize a fixed background mesh which can be Cartesian (Fig. 2(a)), cylindrical, body-fitted (Fig. 2(b)) or unstructured (Fig. 2(c)). We shall identify an interface using the distance function as shown in Fig. 3.

For a fluid-fluid interface with surface tension force at an interface, we shall utilize the continuous surface force model [8] to convert the surface tension force, into a volume force using the distance function. This is then incorporated as volumetric source terms for the momentum equations. We then use the levelset method to model the evolution of the fluid-fluid interface over time. We shall then extend the concept of the continuous surface force model [8], use to model the surface tension force at a fluid-fluid interface, to capture the interfacial conditions for the three different types of interfaces.

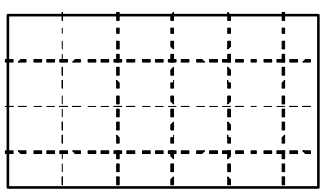

.. $\quad$... (a)

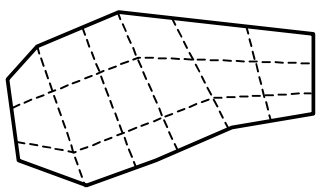

(b)

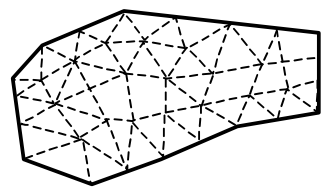

(c)

Figure 2: $\quad$ Three different types of meshes, (a) Cartesian mesh, (b) body-fitted mesh and (c) unstructured mesh.

At a fluid-solid interface, we use the distance function to identify the fluidsolid boundary. We use the concept used in the continuous surface force model to incorporate surface forces at the interface. The translational and rotational motions of the solids are then calculated using the appropriate equations governing the above motions. 

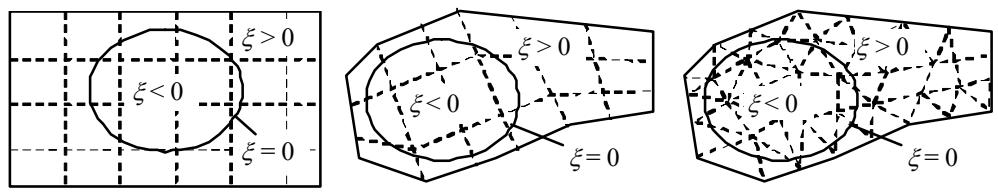

Figure 3: An interface discretization on three different computational meshes.

From Fig. 3, it is clear that an interface is an irregular geometry for the three meshes shown. With this realization, we shall use the distance function to identify a physical boundary. The boundary conditions (e.g. given heat flux, convective heat transfer boundary) at the physical boundary is then incorporated using the continuous surface force concept.

\section{Mathematical formulations}

In this section, we present a brief discussion of the various procedures developed by the authors to model the three different classes of problems. As detailed formulations of the procedures are available in the original articles, repeat presentations of the formulations are not done here. Interested readers are referred to the original articles.

As mentioned above, we use the distance function to identify an interface. Depending on the type of interface, we shall use one of the following three approaches.

\subsection{A fluid-fluid interface}

Once we have identified a fluid-fluid interface using the distance function, we shall model the evolution of the interface using the level-set method of Osher and Sethian [1]. The continuum surface force method [8] is used to model the surface tension forces. We proposed a global mass correction procedure [2] which ensures perfect mass preservation (to machine zero mass errors). Surface tension effects were examined using the proposed method [3]. Phase change effects are modelled using the proposed mass correction procedure [4]. We extended the method to model three-dimensional flow in a square duct [5].

\subsection{A fluid-solid interface}

Similar to the treatment at a fluid-fluid interface, we extend the continuum surface force concept to model forces encountered at a fluid-solid interface [6, 7]. We have model forces due to the electric-double-layer (encountered in microfluidics) and flows of charged particles [10].

We have further extended and combined the two approaches (for fluid-fluid and fluid-solid interfaces) to model the Motion of Particle-Encapsulated Droplets in Microchannels [7]. 


\subsection{Irregular geometries}

As discussed above, droplets and particles are "irregular" geometries for the base mesh as they travel downstream. As a result of this realization, the distance function is used to discretize irregular geometries. A distance-function-base Cartesian coordinate method for irregular geometries called DIFCA was presented by the authors [9]. In this approach, the distance function is used to specify the boundaries of the irregular geometries.

There are two types of interfaces namely, internal interfaces and physical boundaries. Internal interfaces include but are not limited to, (1) interface which separates two materials with different properties, and (2) interface which divides two regions with different source. As the name implies, physical boundaries are interfaces which represent physical interface between the object of interest and the surroundings. Irregular internal interfaces change the discretization equations for internal control volumes. Irregular physical boundaries on the other hand involve changing the discretization equation and incorporation of the boundary conditions into the boundary-adjacent control volumes.

An extension of the continuum surface force approach is formulated to model the three types of boundary conditions namely, (1) given temperature, (2) specified heat flux and (3) convective heat transfer at a boundary. In addition, irregularity due to internal interfaces can also be modelled using this approach. Internal interfaces include but are not limited to, (1) interface which separates two materials with different properties, and (2) interface which divides two regions with different source.

\subsection{Closing remarks}

We formulated an approach to model different "interfaces" using the distance function. The continuum surface force approach and its extensions are then used to model the interface conditions at the various interfaces.

\section{Results and discussions}

Five different problems are discussed in this section to show the capabilities of the approach. More examples can be found in the various articles by the authors $[2-7]$ and is left for the explorations of interested readers.

\subsection{A fluid-fluid interface}

The schematic for a "stratified" two-fluid flow through a double-bend is shown in Fig. 4. The term "stratified" in this case is used loosely for the "stratification" of the fluids on the walls. Both fluids enter the bend with a uniform inlet velocity of $u_{i n, 1}=u_{i n, 2}=u_{i n}$. The initial thickness of fluid 1 at the inlet is $\delta_{i n}$. The length $L$ is twice the width $W$. The Reynolds number Re and the capillary number $\mathrm{Ca}$ are defined as 


$$
\begin{gathered}
\mathrm{Re} \equiv \frac{\rho u_{i n} W}{\mu} \\
C a \equiv \frac{u_{i n} \mu}{\sigma}
\end{gathered}
$$

where $\rho$ is the fluid density, $W$ is the height of the channel, $\mu$ is the fluid viscosity and $\sigma$ is the surface tension. For this example, $\rho_{1} / \rho_{2}, \mu_{1} / \mu_{2}, Q_{1} / Q_{2}$ and $\operatorname{Re}$ (based on fluid 1) are set to 2.0, 2.0, 1.0 and 0.01 respectively. The volumetric flowrate is defined as $Q$.

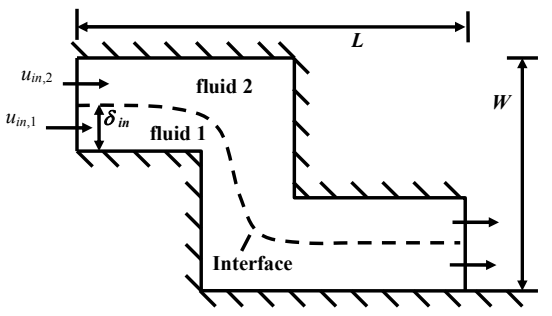

Figure 4: $\quad$ Stratified two-fluid flow through a double-bend.

Fig. 5(a) shows the velocity vectors plot for the solution obtained using $160 \times 80$ control volumes. This fine mesh solution is selected so that small vortices could be detected. Only one in every five velocity vectors is shown to avoid over-crowding. The velocities at the two "boxed" corners are extremely small and hence could not be shown. These regions are enlarged and plotted with the unit velocity vectors. For these enlarged figures, all velocity vectors are shown. It is clear that there is a vortex at each of the corner, an indication of recirculation. Fig. 5(b) shows comparisons between the present prediction and the volume-of-fluid (VOF) method. All the velocity profiles show the variation of $u$ across the streamwise section, but the third velocity profile shows the variation of $v$ instead. These predictions agree well quantitatively for both

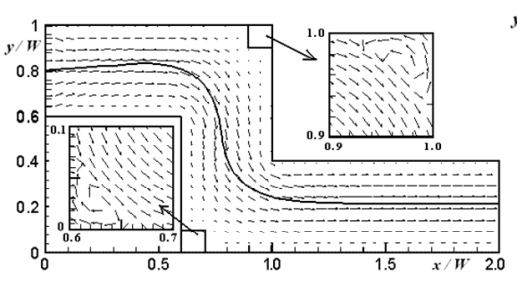

(a)

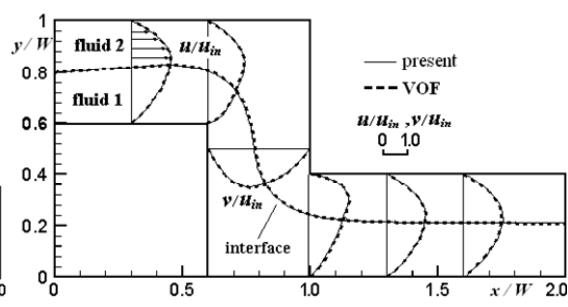

(b)

Figure 5: Stratified two-fluid flow through a double-bend with $C a=0.1$, (a) velocity vectors, (b) the fluid-fluid interface and velocity profile. 
interface location and velocity field. There is no noticeable change in the fluidfluid interface location downstream of around $x / W=1.4$. This implies that the flow is fully-developed. The prediction of VOF is also shown for comparison where reasonable agreement is obtained.

Fig. 6 shows a droplet of fluid 1 suspended in a constricted channel filled with fluid 2. The channel is of length 2 unit. It has a sudden contraction followed by a sudden expansion. At the inlet, the channel height is 1.0 unit. Then, the height contracts abruptly to $W=0.2$ to form a smaller channel of length $L=0.7$, after which it expands to its initial height. For ease of discussion, this smaller channel is henceforth referred as the small channel. The droplet has a diameter of $d_{d}=0.40$ and is initially suspended at $\left(x_{d}, y_{d}\right)=(0.3,0.5)$. The densities and viscosities for fluid 1 are 2 and 2, and for fluid 2 are 1 and 1 respectively. Initially, both fluids are at rest. A stream of fluid 2 then flows into the channel with a uniform velocity of $u_{i n}=0.01$ at the inlet. The droplet is carried by the stream and squeezed through the small channel eventually. Large deformation of the droplet is expected as the droplet has a diameter larger than the size of the small channel.

Generally, the fluid flows faster in the small channel. A lower pressure is developed in the small channel, sucking the droplet into the small channel. This creates a cusp at the leading edge of the droplet. No slip condition is enforced at all walls, including those of the small channel. A typical velocity profile across the small channel for $t=5$ is shown in Fig. 7(a). In the middle of the small channel, the fluid particles flow faster than those near the wall. Since the cusp is driven by a larger velocity at the middle of the channel, it becomes longer and sharper. Once in the small channel, the deformed droplet is squeezed by the main stream through the small channel. At the exit of the small channel, the flow expands since there is a sudden increase in cross sectional area. At this point, the $v$ velocity component is no longer negligible.

The present solution is compared to that of VOF in Figs. 7(a). The spatial and temporal independent VOF solution is obtained using $200 \times 100$ control volumes with $\Delta t=0.10$. The present solutions are in good agreements with that of VOF both qualitatively and quantitatively. The effect of surface tension on the droplet evolution is shown in Fig. 7(b). The surface tension coefficient is set to

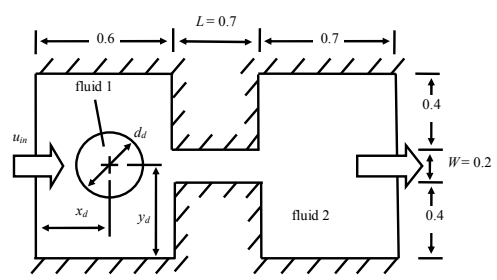

Figure 6: Droplet flow through a constricted channel. 


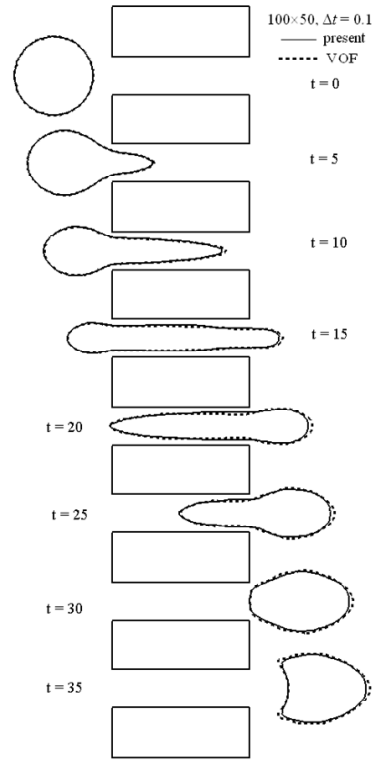

(a)

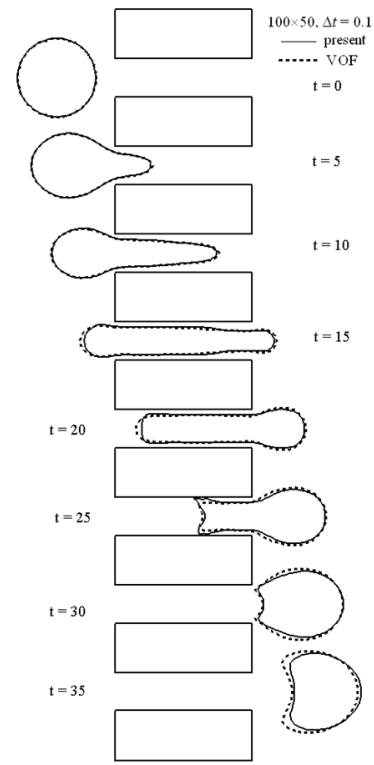

(b)

Figure 7: Droplet flows through a constricted channel (a) $\sigma=0$, (b) $\sigma=0.05$. $\sigma=0.05$. The surface tension force acts to smooth interface with high curvature. In the case of no surface tension (Fig. 7(a)), both the leading cusp and the 'tail' of the droplet are of large curvature, i.e. pointed. When surface tension is present, the pointed nature of both the cusp and 'tail' is suppressed.

\subsection{A fluid-solid interface}

Fig. 8(a) shows the schematic of the problem. A circular cylinder of diameter $d$ is placed at $\left(0, y_{c}\right)$ between two parallel plates spaced $4 d$ apart. The cylinder, which is heavier than the fluid, is initially at rest. When it is released, it travels

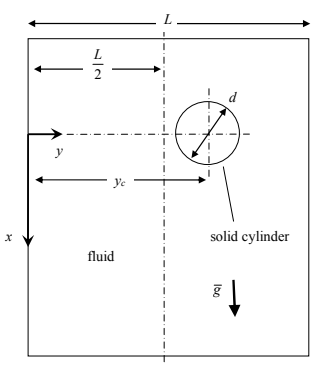

(a)

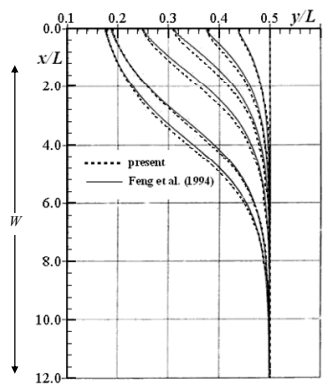

(b)

Figure 8: Sedimentation of a cylindrical particle between parallel plates (a) schematic, (b) particle trajectories for $\mathrm{Re}=0.522$. 
downward due to gravity. It will eventually reach its terminal velocity $u_{T}$. The diameter of the cylinder and its terminal velocity are the characteristic length and the characteristic velocity respectively. The trajectories of the cylinder $(\mathrm{Re}=0.522)$ released at different lateral location off the centerline are shown in Fig. 8(b). The results from the present computation compare well with those reported in the literature [11]. As seen, the cylinder drifts towards the centerline for the Re studied.

\subsection{Irregular geometries}

Figure 9(a) shows a concentric annulus. The inner radius of the annulus is set to 0.5 while the outer radius is specified as 1 . The temperature at the inner radius is set to 1 while the temperature of the cold outer surface is kept at 0 . The $2 \times 2$ domain is discretized into $11 \times 11$ control volumes as shown in Fig. 9(b). The temperature predicted using the staircase method [12] and the proposed method are shown in Figs. 9(c) and 9(d_ respectively. As expected the staircase method does not capture the exact solution as shown in Fig. 9(c). The proposed method on the other hand produces the exact solution! Figure 9(e) shows the temperature as function of radius. The exact solution is reproduced by the proposed method with just 3 nodes in the annulus section.

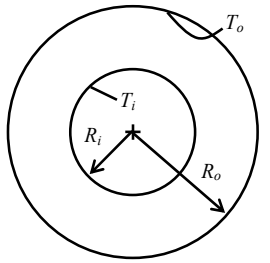

(a)

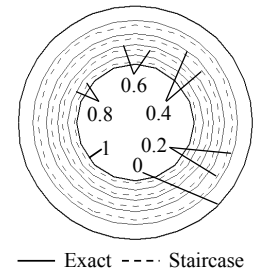

(c)

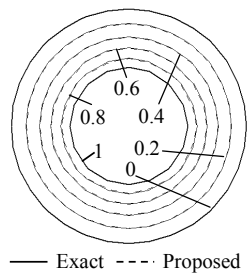

(d)

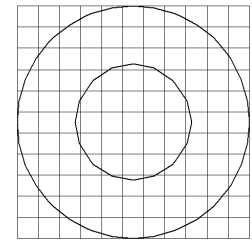

(b)

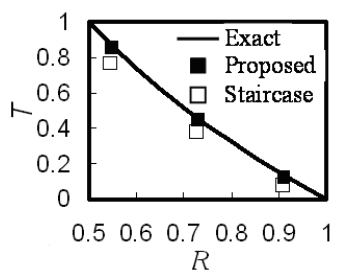

(e)

Figure 9: Conduction in an annulus with specified boundary temperatures and zero source: (a) schematic, (b) computational meshes, (c) temperature contours with staircase method, (d) temperature contours with the proposed method, and (e) temperature distribution. 
As shown in Fig. 10, the curved surface of a semicircle is subjected to a known uniform heat flux of $q$. The temperature of the flat bottom surface is kept at 0 . The radius $R_{o}$ is set to 1 and the thermal conductivity of the semi-circle is set to 1 . A $2 \times 1.2$ rectangular domain is discretized using $41 \times 21$ uniform control volumes as shown in Fig. 10b. The solution is compared with the solution obtained using very fine polar grids. Figure 10(c) shows the dimensionless temperature $\left(T-T_{B}\right) /\left(\dot{q} D_{o}^{2} / 4 k\right)$ comparison between the proposed method and the exact solution. Figure 10(d) shows the comparison of the dimensionless boundary temperature obtained using the proposed method and the exact solution. Excellent agreements have been obtained using the proposed method.

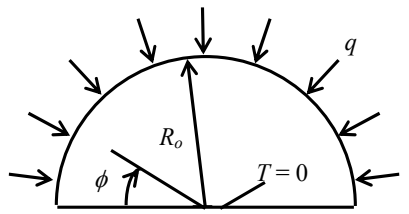

(a)

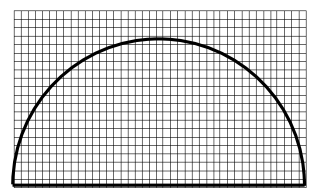

(b)

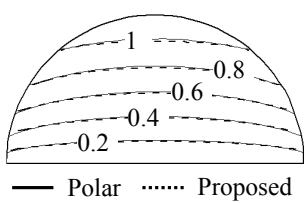

(c)

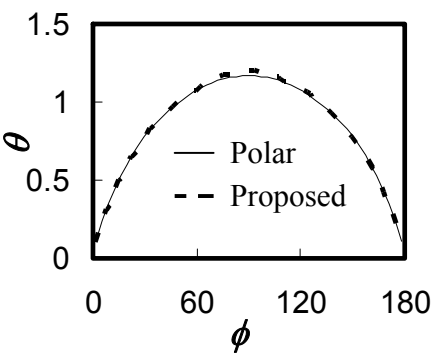

(d)

Figure 10: Conduction in a semi-circular enclosure, (a) schematic, (b) computational meshes, (c) temperature contours, and (d) boundary temperature at the given flux boundary.

\section{Concluding remarks}

We presented five examples problems with different types of interfaces identified using the distance function. The interface conditions for these problems are modelled using the continuum surface force model or its extensions. The results compare well with available published solutions or exact solutions (when available).

\section{References}

[1] Osher, S. \& Sethian, J.A., Fronts Propagating with Curvature-Dependent Speed: Algorithms Based on Hamilton-Jacobi Formulations, Journal of Computational Physics, 79, 12-49, 1988. 
[2] Yap, Y. F., Chai, J. C., Wong, T. N., Toh, K. C., \& Zhang, H. Y., A Global Mass Correction Scheme for the Level-Set Method, Numerical Heat Transfer, Part B., 50(5), 455 - 472, 2006.

[3] Yap, Y. F., Chai, J. C., Wong, T. N., \& Toh, K. C., The Effects of Surface Tension on Two-Dimensional Two-Phase Stratified Flows, Journal of Thermophysics and Heat Transfer, 20(3), 638 - 640, 2006.

[4] Yap, Y. F., Chai, J. C., Toh, K. C., Wong, T. N., \& Lam, Y. C., Numerical Modeling of Unidirectional Stratified Flow with and without Phase Change, International Journal of Heat and Mass Transfer, 48, 477-486, 2005.

[5] Yap, Y. F., Chai, J. C., Toh, K. C., \& Wong, T. N., Modeling the Flows of Two Immiscible Fluids in a Three-Dimensional Square Channel using the Level-Set Method, Numerical Heat Transfer, Part A, 49, 1 - 12, 2006.

[6] Yap Y.F., Chai J.C., Wong T.N., Nguyen K.C., Toh K.C. and Zhang H.Y., Particle transport in microchannels," Numerical Heat Transfer, Part B., 51, 141-157, 2007.

[7] Yap Y.F., Chai J.C., Wong T.N., Nguyen K.C., Toh K.C., Zhang H.Y. \& Yobas, L., A Procedure for the Motion of Particle-Encapsulated Droplets in Microchannels, Numerical Heat Transfer, Part B., 53, 59 - 74, 2008.

[8] Brackbill, J.U., Kothe, D.B. \& Zemach, C., A continuum method for modelling surface tension, Journal of Computational Physics, 100, 335354, 1992.

[9] Chai, J. C., \& Yap, Y. F., A Distance-Function-Based Cartesian (DIFCA) Grid Method for Irregular Geometries, International Journal of Heat and Mass Transfer, 51, 1691 - 1706, 2008.

[10] Wong, T.N., Chai, J.C., Yap, Y.F., Gao, Y., Yang, C. and Ooi, K.T., Electrokinetic two-phase flows, Encyclopedia of Micro- and Nano-Fluidics, edited by D. Li, Springer, 2008.

[11] Feng, J., Hu, H.H. \& Joseph, D.D., Direct simulation of initial value problems for the motion of solid bodies in a Newtonian fluid Part 2: Couette and Poiseuille flows, Journal of Fluid Mechanics, 277, 271-301, 1994.

[12] Patankar, S.V., Numerical Heat Transfer and Fluid Flow, Hemisphere, New York, 1980. 